\title{
The interferometric diameter and internal structure of Sirius A
}

\author{
P. Kervella ${ }^{1}$, F. Thévenin ${ }^{2}$, P. Morel ${ }^{2}$, P. Bordé ${ }^{3}$, and E. Di Folco ${ }^{4}$ \\ 1 European Southern Observatory, Alonso de Cordova 3107, Casilla 19001, Vitacura, Santiago 19, Chile \\ 2 Département Cassini, UMR CNRS 6529, Observatoire de la Côte d'Azur, BP 4229, 06304 Nice Cedex 4, France \\ 3 LESIA, Observatoire de Paris-Meudon, 5 place Jules Janssen, 92195 Meudon Cedex, France \\ ${ }^{4}$ European Southern Observatory, Karl-Schwarzschild-str. 2, 85748 Garching, Germany
}

Received 10 April 2003 / Accepted 23 June 2003

\begin{abstract}
The interferometric observations of dwarf stars in the solar neighbourhood, combined with HIPPARCos parallaxes provide very precise values of their linear diameters. In this paper, we report the direct measurement of the angular diameter of the bright star Sirius A with the VINCI/VLTI instrument. We obtain a uniform disk angular diameter of $\theta_{\mathrm{UD}}=5.936 \pm 0.016$ mas in the $K$ band and a limb darkened value of $\theta_{\mathrm{LD}}=6.039 \pm 0.019$ mas. In combination with the HIPPARCos parallax of $379.22 \pm 1.58$ mas, this translates into a linear diameter of $1.711 \pm 0.013 D_{\odot}$. Using the VINCI/VLTI interferometric diameter and the published properties of Sirius A, we derive internal structure models corresponding to ages between 200 and $250 \pm 12 \mathrm{Myr}$. This range is defined mainly by the hypothesis on the mass of the star, the overshoot and the metallicity.
\end{abstract}

Key words. stars: individual: Sirius - stars: fundamental parameters - stars: evolution - techniques: interferometric

\section{Introduction}

The brightest star in the sky, Sirius A (HR 2491, HD 48915), is an A1V type dwarf. It is also a member of the fifth nearest binary system. The existence of a massive companion, Sirius B, was predicted by Bessel (1844) based on the apparent motion of Sirius A. It was later discovered visually by A. G. Clark in 1862 , and is still today among the most massive white dwarfs (WD) known. Chandrasekhar (1935) published the first model to explain its characteristics. Sirius has been reported several times to be a red star in the ancient times, whereas it is now almost white. The reason behind this color change is still not understood, though several scenarios have been proposed in the last decades (Schlosser \& Bergmann 1985; Bonnet-Bideau \& Gry 1991; Whittet 1999). During the past century, many efforts were directed towards compiling the properties of both stars like their masses or chemical abundances. Sirius A exhibits enhanced metal abundances in its photosphere and has therefore been classified as Am by Abt (1979). As the orbital period is relatively short (50 years), two revolutions of the Sirius A-B binary have already been measured, allowing Gatewood \& Gatewood (1978) to derive precisely the masses of the two components (Sect. 2.1). Moreover, Sirius A has been the first main sequence star to have its diameter measured interferometrically by Hanbury Brown et al. (1967, 1974a).

The chief object in our study is to model the internal structure of Sirius A in order to reproduce its macroscopic characteristics. In this process, we use the angular diameter of this star from our new VINCI/VLTI measurements and the HIPPARCos

Send offprint requests to: P. Kervella, e-mail: pkervell@eso.org parallax to derive its linear diameter. From our models, we compute the age and the initial helium content of Sirius A. We summarize in Sect. 2 the fundamental parameters of this star, while Sects. 3 to 5 are dedicated to the VINCI data processing and analysis. This section includes in particular the fit of the limb darkened disk model to the measured visibilities. Finally, we discuss in Sect. 6 the macroscopic constraints that we apply to our CESAM numerical models (Morel 1997) of the internal structure of Sirius A, and we predict the asteroseismic large frequency spacing of this star.

\section{Fundamental stellar parameters}

\subsection{Masses}

Sirius (A1V + WD) is a visual binary system with a 50 years period that has been studied extensively, and its orbit is known with high precision. From photometric astrometry covering a 60 year period, Gatewood \& Gatewood (1978) determined the orbital photocentric semi-major axis of Sirius A with respect to the system barycenter. This determination relied upon the orbital parameters of the relative visual orbit of Sirius B given by van den Bos (1960). These authors derived a mass for Sirius A of $2.143 \pm 0.056 M_{\odot}$, using a parallax of $\pi=377.7 \pm 3.31$ mas. This precision of $\pi$ has been recently improved thanks to the Hipparcos satellite mission (Perryman et al. 1997) at $\pi=$ $379.22 \pm 1.58$ mas. We note that this value is consistent with the parallax of Van Altena et al. (1995), $\pi=381.6 \pm 2.2$ mas. Consequently, the sum of the masses has to be diminished by $1.2 \%$, giving for Sirius A a lower mass of $2.12 \pm 0.06 M_{\odot}$. Independently of binary orbital elements, Holberg et al. (1998) 
Table 1. Relevant parameters of $\alpha \mathrm{CMa}$ and its calibrators from the literature.

\begin{tabular}{lcccccc}
\hline \hline Name & $\alpha$ CMa & $\theta$ Cen & $\delta$ Lep & $\alpha$ Crt & 31 Ori & HR 4050 \\
HD number & HD 48915 & HD 123139 & HD 39364 & HD 95272 & HD 36167 & HD 89388 \\
\hline$m_{V}$ & -1.47 & 2.06 & 3.80 & 4.07 & 4.71 & 3.38 \\
$m_{K}$ & -1.31 & -0.10 & 1.31 & 1.62 & 0.90 & 0.60 \\
Sp. type & A1V & K0IIIb & G8III/IV & K1III & K5III & K3IIa \\
$T_{\text {eff }}(\mathrm{K})^{a}$ & 9900 & 4980 & 4580 & 4650 & 3930 & 4500 \\
{$[\mathrm{Fe} / \mathrm{H}]^{f}$} & 0.5 & 0.0 & -0.75 & -0.2 & -0.3 & 0.5 \\
$\log g^{a}$ & 4.3 & 2.75 & 2.95 & 2.8 & 1.6 & 1.6 \\
$\pi(\mathrm{mas})^{b}$ & $379.22 \pm 1.58$ & $53.5 \pm 0.8$ & $29.1 \pm 0.6$ & $18.7 \pm 1.0$ & $7.2 \pm 0.8$ & $4.4 \pm 0.5$ \\
$\theta_{\mathrm{LD}}(\mathrm{mas})^{c}$ & $\mathbf{6 . 0 3 9} \pm \mathbf{0 . 0 1 9}^{g}$ & $5.434 \pm 0.020^{e}$ & $2.63 \pm 0.04$ & $2.28 \pm 0.03$ & $3.66 \pm 0.06$ & $5.23 \pm 0.06$ \\
$\theta_{\mathrm{UD}}(\mathrm{mas})^{d}$ & $\mathbf{5 . 9 3 6} \pm \mathbf{0 . 0 1 6}^{g}$ & $5.305 \pm 0.020^{e}$ & $2.57 \pm 0.04$ & $2.22 \pm 0.03$ & $3.55 \pm 0.06$ & $5.09 \pm 0.06$ \\
\hline
\end{tabular}

${ }^{a}$ From Cohen et al. (1992) for $\alpha \mathrm{CMa}$, and from Cayrel de Strobel et al. $(1997,2001)$ for other stars.

${ }^{b}$ Parallaxes from the HipParcos catalogue (Perryman et al. 1997).

$c$ Catalogue values from Cohen et al. (1999) for other stars.

${ }^{d}$ Linear limb darkening coefficients factors from Claret et al. (1995).

e $\theta$ Cen angular diameters from Kervella et al. (2003b).

${ }^{f}$ Qiu et al. for $\alpha$ CMa, and Cayrel at al. (1997) for other stars.

$g$ Sirius angular diameters from this work (see text for details).

have re-determined the mass of Sirius B from precise measurements of its surface gravity and radius. Using the HIPPARcos parallax they found a mass of $M_{B}=1.034 \pm 0.026 M_{\odot}$ in agreement with the revisited sum of the masses. Also based on the HipPARCos parallax, Provencal et al. (1998) have derived a value of $M_{B}=1.000 \pm 0.016 M_{\odot}$, in agreement with Holberg et al. (1998) at a $1.1 \sigma$ level.

\subsection{Age}

The age of the Sirius system has been poorly debated in the literature. Based on Wood's (1995) sequences of cooling age of WD (carbon-oxygen model with thick $\mathrm{H}$ layer), Sirius B is found to be a young WD of about 160 (Holberg et al. 1998) to $210 \pm 20 \mathrm{Myr}$ (Holberg, private communication). The revisited mass of Sirius B $\left(1.034 M_{\odot}\right)$ corresponds to a progenitor mass of $\approx 7 M_{\odot}$, i.e. the approximate mass of a B5 main sequence star, from the empirical initial to final mass relation of Weidemann (2002). Such a massive star evolves to a WD in $40 \pm 5 \mathrm{Myr}$. Consequently, assuming a simultaneous origin of both A \& B components, the Sirius system is expected to be between 200 and $250 \mathrm{Myr}$ old.

\subsection{Spectral properties}

Sirius shows deep metallic spectral lines, and we propose that the corresponding enhancement in metal abundance is a consequence of the strong radiative forces at work in the interior of this star. This hypothesis is supported by the very low rotational velocity (Royer et al. 2002) $v \sin i \approx 16 \mathrm{~km} \mathrm{~s}^{-1}$, that favors the action of these forces. In their comparison with the star Vega, Qiu et al. (2001) concluded that Sirius A appears to be enhanced in abundances by about 1.0 dex on average while $\mathrm{C}$,
$\mathrm{O}, \mathrm{Sc}$ and $\mathrm{Ca}$ are underabundant. In consequence, for such a slow rotator and intermediate mass, it is very hazardous to estimate the mean metal content in the interior of the star from its surface metal abundance.

Therefore, we propose to focus only on the abundances of CNO, that are believed to be little modified by radiative forces (Richer et al. 2000), to estimate the value of $\left(\frac{Z}{X}\right)_{\mathrm{s}}$ at present day. We adopt an average abundance at present day of $\left(\frac{Z}{X}\right)_{\mathrm{s}}=0.012 \pm 0.002$ in our models, that includes the microscopic diffusion of the elements. $\left(\frac{Z}{X}\right)_{i}$ is very important for the internal stellar opacity of the computed model and is a key parameter with respect to the age of the star (see Sect. 6). It results, when using these elements as tracers, that the average metal abundance of Sirius A is in fact about half that of the Sun. While appearing to be low, the adopted $Z_{\mathrm{s}}$ abundance is comparable with Vega's observed value. Vega is a fast rotator and therefore should not be affected by radiative accelerations masking its true metal abundance. This corresponds to an initial value at zero age of $\left(\frac{Z}{X}\right)_{i}=0.0165 \pm 0.002$. In order to validate the previous assumption that the metallicity of outerlayers does not represent the metallicity of the whole star, we also present, in Sect. 6, a model of Sirius A with the observed metallicity $\left(\frac{Z}{X}\right)_{\mathrm{s}}=0.0329$, corresponding to the observed mean surface abundances.

\subsection{Photometry}

The photometric properties of Sirius are summarized in Table 1. The magnitude and the parallax give an intrinsic luminosity of $L / L_{\odot}=25.4 \pm 1.3$. Following Lemke (1989), we adopt an effective temperature of $T_{\text {eff }}=9900 \pm 200 \mathrm{~K}$ and a logarithm of the spectroscopic surface gravity of $\log g=4.3 \pm 0.1$. 


\section{Interferometric observations and data processing}

\subsection{Instrumental setup}

The European Southern Observatory's Very Large Telescope Interferometer (Glindemann et al. 2000) is operated on top of the Cerro Paranal, in Northern Chile since March 2001. For the observations reported in this paper, the light coming from two test siderostats $(0.35 \mathrm{~m}$ aperture) was recombined coherently in VINCI, the VLT INterferometer Commissioning Instrument (Kervella et al. 2000). We used a regular $K$ band filter $(\lambda=$ 2.0-2.4 $\mu \mathrm{m}$ ) but VINCI can also be operated in the $H$ band $(\lambda=1.4-1.8 \mu \mathrm{m})$ using an integrated optics beam combiner (Berger et al. 2001; Kervella et al. 2003a). Two VLTI baselines were used for this program: D1-B3 and E0-G1, respectively 24 and $66 \mathrm{~m}$ in ground length.

\subsection{Data processing}

We used a modified version of the standard VINCI data reduction pipeline (Kervella et al. 2003c), whose general principle is based on the original FLUOR algorithm (Coudé du Foresto et al. 1997). The two calibrated output interferograms are subtracted to remove residual photometric fluctuations. Instead of the classical Fourier analysis, we implemented a timefrequency analysis (Ségransan et al. 1999) based on the continuous wavelet transform (Farge 1992). In this approach, the projection of the signal is not done onto a sine wave (Fourier transform), but on a function, i.e. the wavelet, that is localized in both time and frequency. We used as a basis the Morlet wavelet, a Gaussian envelope multiplied by a sine wave. With the proper choice of the number of oscillations inside the Gaussian envelope, this wavelet closely matches a VINCI interferogram. It is therefore very efficient at localizing the signal in both time and frequency.

The differential piston corrupts the amplitude and the shape of the fringe peak in the wavelets power spectrum. A selection based on the shape of fringe peak in the time-frequency domain is used to remove "pistonned" and false detection interferograms. Squared coherence factors $\mu^{2}$ are then derived by integrating the wavelet power spectral density (PSD) of the interferograms at the position and frequency of the fringes. The residual photon and detector noise are removed by making a least squares fit of the PSD at high and low frequency. The resulting measurement stability is satisfactory: on a good series of 500 interferograms of Sirius $(10 \mathrm{~min})$ the standard deviation is typically $2.0 \%$, and the final bootstrapped statistical error on the average $\mu$ is only $0.16 \%$. The $\mu^{2}$ values are converted into calibrated visibilities $V^{2}$ through the observation of calibrator stars (Sect. 4).

\section{Calibration}

The calibration of Sirius visibilities was achieved using wellknown calibrator stars that have been selected in the Cohen et al. (1999) catalogue. The characteristics of the selected calibrators are listed in Table 1. The limb-darkened disk (LD) angular diameter of these stars was converted into a uniform disk value using linear coefficients taken from Claret et al. (1995). As demonstrated by Bordé et al. (2002), the star diameters in the Cohen et al. (1999) list have been measured very homogeneously to a relative precision of approximately $1 \%$ and agree well with other angular diameter estimation methods. The interferometric efficiency is computed from the coherence factors obtained on these calibrators, taking into account the bandwidth smearing effect (Sect. 5.2) and a uniform disk angular diameter model. This process yields the calibrated squared visibilities that are used for the model fit.

In Table 2, two error bars are given for each $V^{2}$ value:

- one statistical error bar, computed from the dispersion of the visibility values obtained during the observation,

- one systematic error bar defined by the uncertainty on the knowledge of the calibrator stars angular sizes.

While the statistical error can be diminished by repeatedly observing the target, the systematic error cannot be reduced by averaging measurements obtained using the same calibrator. This is taken into account in our model fitting by checking a posteriori that the resulting uncertainty of the model visibility is larger than the systematic errors of each measured visibility value. This conservative approach ensures that we are not underestimating the final error bars on the angular diameter of Sirius.

\section{Angular diameter computation}

\subsection{Intensity profile}

From the visibility curve shape before the first minimum, it is almost impossible to distinguish between a uniform disk (UD) and limb darkened (LD) model. Therefore, it is necessary to use a model of the stellar disk limb darkening to deduce the photospheric angular size of the star, from the observed visibility values. The intensity profiles that we used were computed by Claret (2000), based on model atmospheres by Kurucz (1992). We chose the four parameters $I(\mu) / I(1)$ law of this author, where $\mu=\cos \theta$ is the cosine of the azimuth of a surface element of the star. They are accurate approximations of the numerical results from the ATLAS modeling code.

To compare the different types of approximated limb darkening laws, we chose the following approximate parameters for Sirius: $T_{\text {eff }}=10000 \mathrm{~K}$ (for Claret et al. 1995 and Claret 2000) or $T_{\text {eff }}=9800 \mathrm{~K}$ (for Claret 1998), $\log g=4.0,[\mathrm{Fe} / \mathrm{H}]=+0.5$, $V_{\text {turb }}=0 \mathrm{~km} \mathrm{~s}^{-1}$. These were chosen as close as possible to the Cohen et al. (1992) values. As noted by Claret (2000), the sensitivity of the limb darkening of a $T_{\text {eff }}=10000 \mathrm{~K}$ star to the metallicity is negligible, so the true metal content of Sirius is not critical. Different $I(\mu) / I(1)$ square root and four parameters laws for Sirius, from Claret et al. (1995) and Claret (1998, 2000) are plotted in Fig. 3. Though no formal error bars are provided for these limb darkening models, the small differences between the four curves demonstrates the good internal consistency of the different types of laws.

The limb darkening is directly measurable by interferometry beyond the first minimum of the visibility function, as demonstrated by several authors on giant stars 
Table 2. Sirius squared visibilities.

\begin{tabular}{lccccl}
\hline \hline JD & Stations & Baseline $(\mathrm{m})$ & Az. $^{b}$ & $V^{2} \pm$ Stat. \pm Syst. & Calibrators $^{a}$ \\
\hline 2452682.71653 & D1-B3 & 17.758 & 81.68 & $0.8602 \pm 0.0206 \pm 0.0007$ & $\delta$ Lep, $\alpha$ Crt \\
2452682.71251 & D1-B3 & 18.139 & 81.45 & $0.8742 \pm 0.0210 \pm 0.0007$ & $\delta$ Lep, $\alpha$ Crt \\
2452679.71932 & D1-B3 & 18.270 & 81.36 & $0.8769 \pm 0.0313 \pm 0.0023$ & $\delta$ Lep, 31 Ori, HR 4050 \\
2452682.70813 & D1-B3 & 18.544 & 81.18 & $0.8668 \pm 0.0208 \pm 0.0007$ & $\delta$ Lep, $\alpha$ Crt \\
2452679.71478 & D1-B3 & 18.684 & 81.08 & $0.8748 \pm 0.0309 \pm 0.0023$ & $\delta$ Lep, 31 Ori, HR 4050 \\
2452679.71085 & D1-B3 & 19.032 & 80.84 & $0.8627 \pm 0.0300 \pm 0.0022$ & $\delta$ Lep, 31 Ori, HR 4050 \\
2452679.66856 & D1-B3 & 22.063 & 78.01 & $0.8092 \pm 0.0280 \pm 0.0021$ & $\delta$ Lep, 31 Ori, HR 4050 \\
2452679.66415 & D1-B3 & 22.301 & 77.68 & $0.8110 \pm 0.0281 \pm 0.0021$ & $\delta$ Lep, 31 Ori, HR 4050 \\
2452679.65967 & D1-B3 & 22.527 & 77.35 & $0.8048 \pm 0.0278 \pm 0.0021$ & $\delta$ Lep, 31 Ori, HR 4050 \\
2452361.62629 & E0-G1 & 60.439 & 1.29 & $0.1599 \pm 0.0074 \pm 0.0045$ & $\theta$ Cen \\
2452361.64154 & E0-G1 & 60.574 & 5.16 & $0.1608 \pm 0.0062 \pm 0.0045$ & $\theta$ Cen \\
2452361.57907 & E0-G1 & 61.038 & 169.41 & $0.1472 \pm 0.0085 \pm 0.0041$ & $\theta$ Cen \\
2452358.55861 & E0-G1 & 62.027 & 162.72 & $0.1433 \pm 0.0062 \pm 0.0042$ & $\theta$ Cen \\
2452340.60484 & E0-G1 & 62.148 & 162.07 & $0.1311 \pm 0.0059 \pm 0.0014$ & $\theta$ Cen \\
2452340.57040 & E0-G1 & 63.651 & 155.00 & $0.1243 \pm 0.0063 \pm 0.0014$ & $\theta$ Cen \\
2452340.56629 & E0-G1 & 63.831 & 154.23 & $0.1226 \pm 0.0054 \pm 0.0013$ & $\theta$ Cen \\
2452340.54577 & E0-G1 & 64.678 & 150.65 & $0.1186 \pm 0.0051 \pm 0.0013$ & $\theta$ Cen \\
2452340.54210 & E0-G1 & 64.816 & 150.06 & $0.1152 \pm 0.0052 \pm 0.0013$ & $\theta$ Cen \\
\hline
\end{tabular}

a All calibrators for Sirius were chosen in the Cohen et al. (1999) catalogue.

$b$ The azimuth is counted clockwise from North, in degrees ( $E=90 \mathrm{deg})$.

(Quirrenbach et al. 1996; Wittkowski et al. 2001). Unfortunately, for stars of the angular size of Sirius observed in the $K$ band, this requires baseline of 80 to $120 \mathrm{~m}$ that were not available for the measurements reported here. It is intended in the near future to measure directly the LD of a number of nearby stars, using the VINCI and AMBER (Petrov et al. 2000) instruments on the long baselines of the VLTI. With lengths of up to $202 \mathrm{~m}$, they will allow the exploration of the secondary and higher order lobes of the visibility function.

\subsection{Visibility model}

The VINCI instrument bandpass corresponds to the $K$ band filter $(2-2.4 \mu \mathrm{m})$. An important effect of this relatively large spectral bandwidth is that several spatial frequencies are simultaneously observed by the interferometer. This effect is known as the bandwidth smearing. It is usually negligible for $V^{2} \geq 40 \%$, but this is not the case when visibilities come closer to the first minimum of the visibility function. In the case of Sirius A observed with the E0-G1 baseline, $V^{2}$ is already low (13\%) and we have to consider it.

To account for the bandwidth smearing, the model visibility is computed for regularly spaced wavenumber spectral bins over the $K$ band, and then integrated to obtain the model visibility. We assume in this paper a limb darkening model following the four parameters law of Claret (2000):

$I(\mu) / I(1)=1-\sum_{k=1}^{4} a_{k}\left(1-\mu^{\frac{k}{2}}\right)$
The $a_{k}$ coefficient are tabulated by this author for a wide range of stellar parameters $\left(T_{\text {eff }}, \log g, \ldots\right)$ and photometric bands $(U$ to $K)$. For Sirius $\left(V_{\mathrm{T}}=0 \mathrm{~km} \mathrm{~s}^{-1}, T_{\text {eff }}=10000 \mathrm{~K}, \log g=4.5\right.$, $[\mathrm{M} / \mathrm{H}]=0)$ we find $a_{1}=0.5318, a_{2}=-0.6921, a_{3}=0.6698$, and $a_{4}=-0.2431$. Figure 3 shows the corresponding intensity profile $I(\mu) / I(1)$. In this paper, we assume that the limb darkening law does not change over the $K$ band, which is reasonable for a hot and compact stellar atmosphere, but this computation can easily be extended to a wavenumber dependent $I(\mu, \sigma)$ intensity profile. Following Davis et al. (2000), using a Hankel integral, we can derive the visibility law $V\left(B, \theta_{\mathrm{LD}}, \sigma\right)$ from the intensity profile:

$V=\frac{1}{A} \int_{0}^{1} I(\mu) J_{0}\left(\pi B \sigma \theta_{\mathrm{LD}} \sqrt{1-\mu^{2}}\right) \mu \mathrm{d} \mu$

where $\sigma$ is the wavenumber:

$\sigma=1 / \lambda$

and $A$ is a normalization factor:

$A=\int_{0}^{1} I(\mu) \mu \mathrm{d} \mu$.

The integral of the binned squared visibilities is computed numerically over the $K$ band and gives the model $V^{2}$ for the projected baseline $B$ and the angular diameter $\theta_{\mathrm{LD}}$ through the relation:

$V^{2}\left(\theta_{\mathrm{LD}}, B\right)=\int_{K}\left[V\left(B, \theta_{\mathrm{LD}}, \sigma\right) T(\sigma)\right]^{2} \mathrm{~d} \sigma$ 


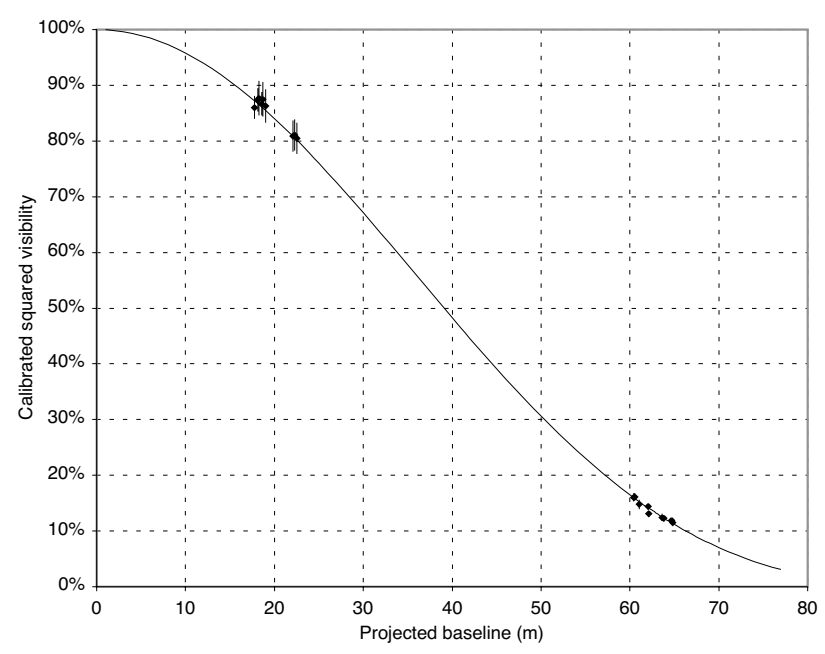

Fig. 1. Overview of the VINCI/VLTI visibility measurements obtained on Sirius. The solid line is the best fit visibility model, that takes into account the limb darkening and the bandwidth smearing.

where $T(\sigma)$ is the normalized instrumental transmission defined so that

$\int_{K} T(\sigma) \mathrm{d} \sigma=1$

We computed a model of $T(\sigma)$ by taking into account the instrumental transmission of VINCI and the VLTI. It was first estimated by considering all known factors (filter, fibers, atmospheric transmission, ...) and then calibrated on sky based on several observations of bright stars with the $8 \mathrm{~m}$ UTs (see Kervella et al. 2003b for more details). This gives, for Sirius, an average measurement wavelength of $2.176 \mu \mathrm{m}$.

The $V^{2}\left(\theta_{\mathrm{LD}}, B\right)$ model is adjusted numerically to the observed $\left(B, V^{2}\right)$ data using a classical $\chi^{2}$ minimization process to derive $\theta_{\mathrm{LD}}$. The reduced $\chi^{2}$ of the global fit is 0.30 , characteristic of a satisfactory correspondence between the model and the measured $V^{2}$ values. Figures 1 and 2 show the position of the VINCI points on the squared visibility curve of the chosen model.

\subsection{Angular and linear diameters}

The $\chi^{2}$ minimization gives a limb darkened angular diameter $\theta_{\mathrm{LD}}=6.039 \pm 0.019$ mas for Sirius $\mathrm{A}$, while a simpler uniform disk model yields $\theta_{\mathrm{UD}}=5.936 \pm 0.016$ mas. The conversion formula between the LD angular diameter and the linear diameter $D$ (in solar unit) is:

$D=\frac{\theta_{\mathrm{LD}}}{2 p \tan \left(\theta_{\odot} / 2\right)}$.

Assuming a solar angular radius of $\theta_{\odot} / 2=959.64 \pm 0.02^{\prime \prime}$ (Chollet \& Sinceac 1999) and the HiPPARcos parallax $p=$ $379.22 \pm 1.58$ mas, we obtain $D_{\text {Sirius }}=1.711 \pm 0.013 D_{\odot}$. It should be noted that the parallax uncertainty is largely dominating the final error on the linear diameter. Therefore, an intrinsically more precise interferometric measurement would not result in an improvement of the linear diameter precision. The

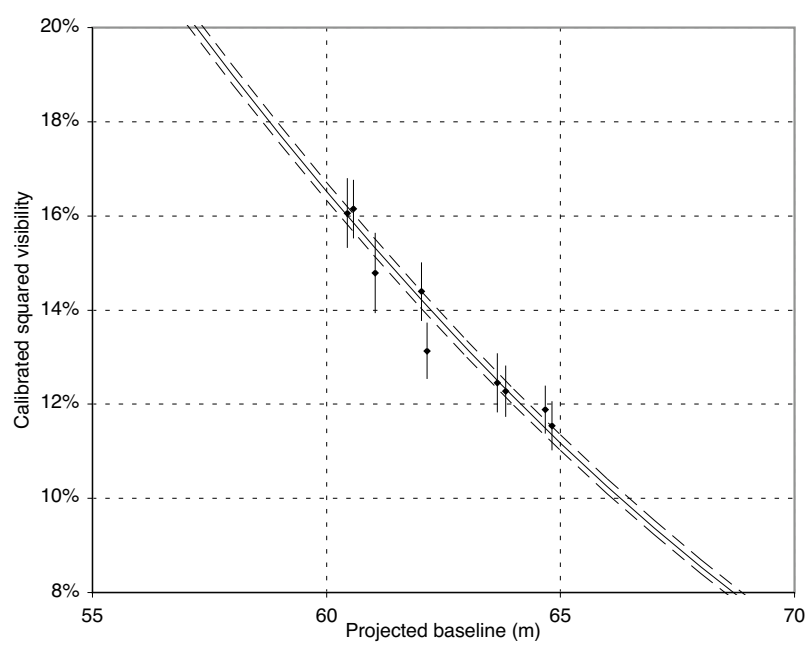

Fig. 2. Visibility measurements obtained on Sirius on the E0-G1 baseline. The thin line is the best fit model, and the dashed lines mark the limits of the $1 \sigma$ error domain. For clarity, the plotted error bars are the quadratic sum of the statistical and systematic errors.

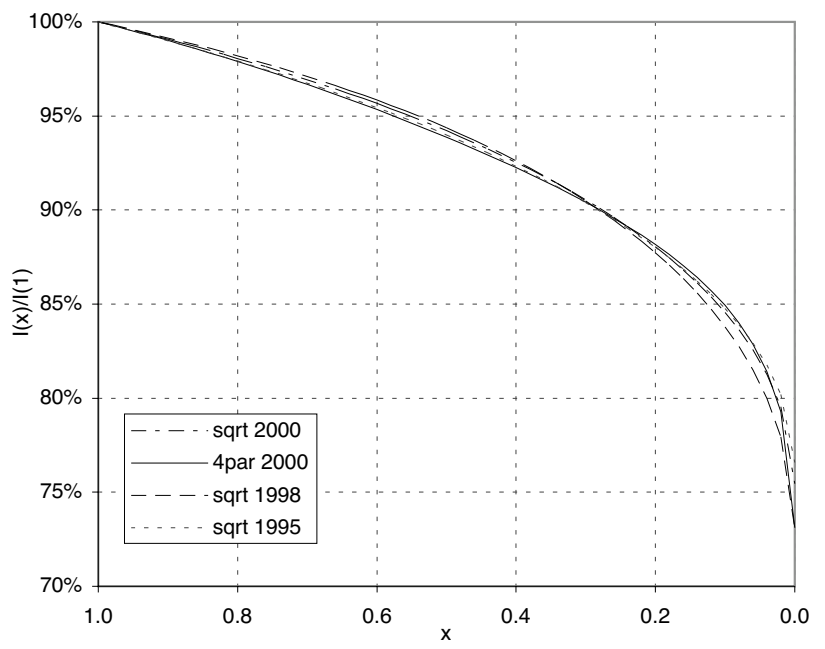

Fig. 3. Published LD laws for Sirius in the $K$ band, from Claret et al. (1995), Claret (1998) and Claret (2000). The square root law is already a good approximation to the Kurucz atmosphere models. The solid line shows results with the most recent four parameters law introduced by Claret (2000). This last version was chosen for the Sirius LD fit of this paper.

contribution of the VINCI/VLTI measurement uncertainty in the error bar is only $\pm 0.004 D_{\odot}$, or $37 \%$ of the total error.

\subsection{Discussion}

Our LD angular diameter value can be compared to the value published by Hanbury Brown et al. (1974a). They found, using the Narrabri intensity interferometer, $\theta_{\mathrm{LD}}=5.89 \pm 0.16$ mas. The difference with our value of $\theta_{\mathrm{LD}}=6.039 \pm 0.019$ mas is $-0.8 \sigma$, making the two results statistically compatible within their error bars. 
Davis \& Tango (1986) have obtained a value of $\theta_{\mathrm{UD}}=$ $5.63 \pm 0.08$ mas using the amplitude interferometer, and revised the Hanbury Brown et al. (1974a) value to $\theta_{\text {UD }}=5.60 \pm$ 0.07 mas. Using a limb darkening coefficient $u=0.5900$ ( $B$ band from Claret et al. 2000) and the conversion factor formula from Hanbury Brown et al. (1974b):

$\rho=\frac{\theta_{\mathrm{LD}}}{\theta_{\mathrm{UD}}}=\sqrt{\frac{1-u / 3}{1-7 u / 15}}$

we find $\rho=1.053$. This translates into $\theta_{\mathrm{LD}}=5.93 \pm 0.08$ mas and $\theta_{\mathrm{LD}}=5.90 \pm 0.07$ mas for the above UD values, respectively. They differ respectively by -1.3 and $-1.9 \sigma$ from our result. This difference, marginally significant, could be explained by an uncertainty on the limb darkening factor. It is much stronger at visible wavelengths than in the infrared, and can be affected by the presence of spectral features (Tango \& Davis 2002).

Using spectro-photometric observations, Cohen et al. (1992) have derived the angular diameter of Sirius and found 6.04 mas (with Kurucz model atmospheres), in remarkable agreement with our direct measurement.

Due to the relatively slow rotational velocity of Sirius ( $v \sin i \approx 16 \mathrm{~km} \mathrm{~s}^{-1}$, from Royer et al. 2002), we do not expect any detectable flattening of its disk. In addition, the flux of Sirius B is totally negligible compared to A, in particular in the $K$ band. We therefore do not foresee an asymmetry of the visibility function in azimuth due to these two contributors.

Hanbury Brown et al. (1974a) have observed a small asymmetry of the Sirius visibility function, proposing tentatively that this may come from a dust disk around this star. In the error bars, our measurements do not show this asymmetry, as the D1-C3 and E0-G1 points are in good agreement with our single disk visibility model. Nevertheless, it should be noted that we have probed only two azimuth values, namely 80 and 160 degrees (D1-C3 and E0-G1 baselines, respectively), and that our error bars on the diameter measurements on the shorter baseline are relatively large. Therefore, we cannot exclude formally the presence of a disk accounting for approximately $1 \%$ of the stellar flux.

As a remark, Benest \& Duvent (1995) have proposed that a late M5 dwarf could be orbiting Sirius A, but our measurements do not have the necessary sensitivity to record the visibility modulation that would be produced by a star of this type, with $m_{K} \geq 5$.

\section{Modeling}

\subsection{Hypothesis}

We computed a number of evolutionary models of Sirius A using the CESAM code (Morel 1997) including the pre-main sequence evolution. The ordinary assumptions of stellar modeling are made, i.e. spherical symmetry, no rotation, no magnetic field, no mass loss. The relevant nuclear reaction rates are taken from the NACRE compilation (Angulo et al. 1999). The equation of state adopted is EFF (Eggleton et al. 1973), and the OPAL opacities are from Iglesias \& Rogers (1996) with the
Grevesse \& Noels (1993) mixture. The microscopic diffusion is described using the formalism of Burgers (1969) with the resistance coefficients of Paquette et al. (1986). We take into account the radiative diffusivity as recommended by Morel \& Thévenin (2002), that limits the efficiency of the microscopic diffusion in outerlayers of stars with intermediate masses. We have neglected the radiative accelerations, as well as the changes of abundance ratios between metals within $Z$. The atmosphere is restored using Hopf's $T(\tau)$ law (Mihalas 1978). The adopted radius of the star is the bolometric radius, where $T\left(\tau_{\star}\right)=T_{\text {eff }}$. In the convection zones the temperature gradient is computed according to the MLT $_{\mathrm{CM}}$ convection theory with a mixing length parameter of $\Lambda=1$ (Canuto \& Mazzitelli 1991, 1992). Following the prescriptions of Schaller et al. (1992) we have computed models that include overshooting of the convective core (radius $\left.R_{\mathrm{co}}\right)$ over the distance $O_{\mathrm{v}}=A \min \left(H_{\mathrm{p}}, R_{\mathrm{co}}\right)$. We adopted a value $A=0.15$ in agreement with the results by Ribas et al. (2000) for a $2 M_{\odot}$ star. In addition, we have also computed stellar models without overshooting $(A=0.00)$ of the convective core and with $A=0.20$ to estimate the effect of this parameter on the age of the star. As a comparison, we have also built a model with enhanced $Z_{\mathrm{s}} / X$, corresponding to the atmospheric abundances published by Qiu et al. (2001). It is intended to demonstrate the strong effect of this overestimated value of the internal abundance on the evolution stage of Sirius. Each model is described by about 760 mass shells. We considered that our model was representative of Sirius A when we reached the interferometric radius and the observed luminosity and effective temperature of this star (within their respective error bars). The main characteristics of the computed models are presented in Table 3.

\subsection{Results and discussion}

The HR evolution diagram is presented in Fig. 4. We adopt the standard definition of the ZAMS, i.e. where nuclear reactions begin to dominate gravitation as the primary source of energy by at least $50 \%$. For the mass of Sirius A this corresponds to an elapsed time of 4.2 Myr. Note that $100 \%$ of energy comes from nuclear reactions only after $10 \mathrm{Myr}$. The evolution of models starts with the quasi-static contraction of a cloud with a central temperature of 0.5 MK. According to these definitions, our model $b$ of Sirius, which fits the VINCI/VLTI radius and satisfies the adopted luminosity and $T_{\text {eff }}$, is aged of $197 \pm 12 \mathrm{Myr}$. The error bar is fixed by the uncertainty on the VINCI/VLTI radius, that is itself largely dominated by the HIPPARCos parallax error bar.

Because the age of the WD can reach 210 Myr (Holberg, private communication) we also computed models with a lower mass $2.07 M_{\odot}$ which fit both the observed radius and the $T_{\text {eff }}$. We had to change sligthly $Y_{\mathrm{i}}$ and the value of $\left(\frac{Z}{X}\right)_{\mathrm{i}}$ within its error bar, see model $d$, resulting in an age of $243 \mathrm{Myr}$ for Sirius. We can produce a greater age of $250 \mathrm{Myr}(\operatorname{model} e)$ when increasing the overshooting parameter A to the value $A=0.20$. At the end of the pre-main sequence, Sirius A has generated a convective core due to the equilibration of the CNO nuclear cycle. At the expected age of Sirius A for model $d$ the 
Table 3. Characteristics of our $\alpha \mathrm{CMa}$ A models remaining within the uncertainty box of the observed properties of this star. The subscripts $i$ and $s$ refer respectively to the initial content at zero age and the surface content at the end of the computed evolution. The adopted luminosity is $\log \left(L / L_{\odot}\right)=1.405 \pm 0.022$ with $T_{\text {eff }}=9900 \pm 200 \mathrm{~K}$. The observed abundance of oxygen is $-0.32 \pm 0.20 \mathrm{dex}$ (see text). The precision of the age is $\pm 12 \mathrm{Myr}$ corresponding to the crossing time of the shaded parallelogram.

\begin{tabular}{lccccc}
\hline \hline Model & $\mathrm{a}$ & $\mathrm{b}$ & $\mathrm{c}$ & $\mathrm{d}$ & $\mathrm{e}$ \\
\hline$M / M_{\odot}$ & 2.12 & 2.12 & 2.12 & 2.07 & 2.07 \\
age $(\mathrm{Myr})$ & 180 & 197 & 25 & 243 & 250 \\
overshoot & 0.00 & 0.15 & 0.15 & 0.15 & 0.20 \\
$T_{\text {eff }}(\mathrm{K})$ & 9870 & 9906 & 9817 & 9936 & 9944 \\
$\log \left(L / L_{\odot}\right)$ & 1.398 & 1.403 & 1.385 & 1.408 & 1.409 \\
$\log g$ & 4.30 & 4.29 & 4.30 & 4.29 & 4.29 \\
$Y_{\mathrm{i}}$ & 0.267 & 0.267 & 0.330 & 0.273 & 0.273 \\
$\left(\frac{Z}{X}\right)_{\mathrm{i}}$ & 0.0165 & 0.0165 & 0.0350 & 0.0145 & 0.0145 \\
$\left(\frac{Z}{X}\right)_{\mathrm{s}}$ & 0.0116 & 0.0115 & 0.0289 & 0.0099 & 0.0099 \\
{$[\mathrm{O} / \mathrm{H}]_{\mathrm{i}}$} & -0.17 & -0.17 & & -0.23 & -0.23 \\
{$[\mathrm{O} / \mathrm{H}]_{\mathrm{s}}$} & -0.33 & -0.33 & & -0.39 & -0.39 \\
$R / R_{\odot}$ & 1.7111 & 1.7107 & 1.7060 & 1.7105 & 1.7103 \\
$\overline{\Delta v_{0}}(\mu \mathrm{Hz})$ & 82 & 82 & 82 & 81 & 81 \\
\hline
\end{tabular}

hydrogen mass fraction at center is $X_{\mathrm{c}}=0.590$ with a temperature of $22.46 \times 10^{6} \mathrm{~K}$. The base of the external convective zone is situated at $0.9926 R_{\star}$ and $Y_{\mathrm{s}}=0.200$.

Removing the overshooting from our model reduces the age of the binary by $10 \%$ (model $a$ ) while the model $c$ with enhanced abundances leads to a too young age (25 Myr) for Sirius A, in significant disagreement with the age of Sirius B. At the present status of our observational constraints on Sirius A, it is impossible to decide if overshoot exists or not in the central convective core. Note that our model gives a surface abundance of $[\mathrm{M} / \mathrm{H}]_{\odot}^{\text {Sirius }}=-0.32$ dex which agrees with the abundance of the oxygen found by Kamp et al. (2002). Finally, if Sirius A is able to excite radial modes of oscillation, we predict the mean large frequency spacing $\overline{\Delta v_{0}}$ to be ranging between 81 and $82 \mu \mathrm{Hz}$ This is the primary observable of asteroseismology, and corresponds to the difference between the frequencies of oscillation modes with consecutive radial order $n$ (see Table 3). This large spacing is sensitive primarily to the stellar density. It could therefore give a direct, high accuracy estimation of the mass of Sirius A when combined with the observed radius reported in this paper.

\section{Conclusion and perspectives}

We have reported in this paper our long-baseline interferometric observations of $\alpha$ CMa A using the VINCI/VLTI instrument. In conjunction with constraints from previous spectrophotometric observations, we derived an evolutionary model for Sirius A. We propose that the apparently high surface metallic content of Sirius is not characteristic of the whole average value of $Z$ for the star, and is caused by the levitation

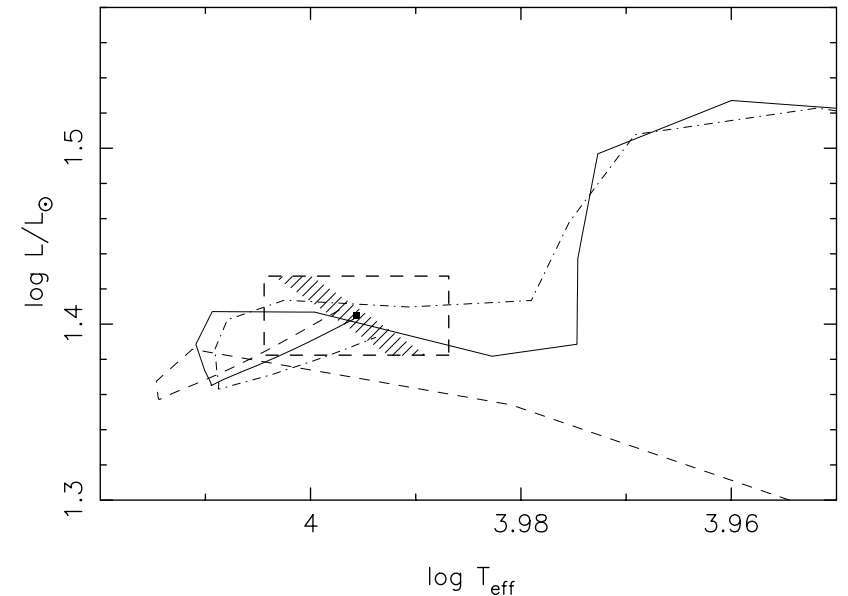

Fig. 4. Evolutionary tracks in the HR diagram of models of Sirius A. The dashed rectangle delimits the uncertainty domain in luminosity and effective temperature, while the shaded area represents the uncertainty on the interferometric radius. The continuous line corresponds to model $b$ with overshoot and the dashed-dot line to model $a$ without overshoot, both with a mass of $2.12 M_{\odot}$. Model $d$, corresponding to $M=2.07 M_{\odot}$ and an age of $243 \mathrm{Myr}$, is represented by a dashed line. The filled square is the center of the rectangle.

of the heavy elements on the thin upper convective layer of Sirius A. By means of our model, we derive an age of 200 to $250 \pm 12 \mathrm{Myr}$, consistent with the two evolutionary time estimates of Sirius B. The oldest age of Sirius A corresponds to a lower mass of $2.07 M_{\odot}$ which is the lowest mass value acceptable within its error bar.

The accuracy on this age is greatly strengthened by the VINCI/VLTI radius, thus encouraging further studies to improve our knowledge of the diameter of nearby stars. Based on our model, we predict that the asteroseismic large frequency spacing of Sirius A should be 81 to $82 \mu \mathrm{Hz}$, if it exhibits radial oscillations. A more complete modeling of the external layers of this star for asterosismic frequencies prediction, including radiative accelerations, is currently in preparation.

We would like to emphasize here the need for very high accuracy parallax values, beyond the HIPPARcos precision. We have shown that the uncertainty on our determination of the linear radius of Sirius A is largely dominated by the error on the distance, despite its proximity $(2.7 \mathrm{pc})$. The next generation of interferometric instruments, such as the AMBER beam combiner (Petrov et al. 2000), will provide high precision angular diameters, together with their wavelength dependence. In order to constrain the stellar structure models, that output linear values, it will be necessary to match the precision on the distance to the angular diameter uncertainty. It is expected that future space based astrometric missions will be able to give a significant improvement over the HIPPARcos parallaxes, and are therefore crucial in this respect. This work on Sirius A and the study of $\alpha$ Cen A \& B by Kervella et al. (2003b) have demonstrated that for the nearest stars, thanks to the very small error bar on the linear radius, we have advantage to replace the classical error bars in the HR diagram, e.g. luminosity and effective temperature, by the couple luminosity and radius. 
The direct measurement of the limb darkening of Sirius A is the next step of the interferometric study of this star. It will allow a refined modeling of its atmosphere and will complete the calibration of the radius that we presented in this paper. This observation will be achieved soon using the long baselines of the VLTI.

Acknowledgements. These interferometric measurements have been obtained using the VINCI instrument installed at the VLTI. The VLTI is operated by the European Southern Observatory at Cerro Paranal, Chile. This work has made use of the wavelets data processing technique, developed by D. Ségransan (Observatoire de Genève) and integrated in the VINCI pipeline. Observations with the VLTI are only made possible through the efforts of the VLTI team, for which we are grateful. The VINCI public commissioning data reported in this paper have been retrieved from the ESO/ST-ECF Archive (Garching, Germany). This research has made use of the SIMBAD database at CDS, Strasbourg (France).

\section{References}

Abt, H. A. 1979, AJ, 84, 1591

Angulo, C., Arnould, M., Rayet, M., et al. 1999, Nucl. Phys. A, 656, 3

Benest, D., \& Duvent, J.-L. 1995, A\&A, 299, 621

Berger, J.-P., Haguenauer, P., Kern, P. et al. 2001, A\&A, 376, L31

Bessel, F. W. 1844, MNRAS, 6, 136

Bonnet-Bideau, J. M., \& Gry, C. 1991, A\&A, 252, 193

Bordé, P., Coudé du Foresto, V., Chagnon, G., \& Perrin, G. 2002, A\&A, 393, 183

Burgers, J. M. 1969, Flow Equations for Composite Gases (New York: Academic Press)

Canuto, V. M., \& Mazitelli, I. 1991, ApJ, 370, 295

Canuto, V. M., \& Mazitelli, I. 1992, ApJ, 389, 729

Cayrel de Strobel, G., Soubiran, C., Friel, E. D., Ralite, N., \& Francois, P. 1997, A\&AS, 124, 299

Cayrel de Strobel, G., Soubiran, C., \& Ralite, N. 2001, A\&A, 373, 159

Chandrasekhar, S. 1935, MNRAS, 95, 207

Chollet, F., \& Sinceac, V. 1999, A\&AS, 139, 219

Claret, A., Diaz-Cordovez, J., \& Gimenez, A. 1995, A\&AS, 114, 247

Claret, A. 1998, A\&A, 335, 647

Claret, A. 2000, A\&A, 363, 1081

Cohen, M., Walker, R. G., Barlow, M. J., \& Deacon, J. R. 1992, AJ, 104,1650

Cohen, M., Walker, R. G., Carter, B., et al. 1999, AJ, 117, 1864

Coudé du Foresto, V., Ridgway, S., \& Mariotti, J.-M. 1997, A\&AS, 121,379

Davis, J., \& Tango, W. J. 1986, Nature, 323, 234

Davis, J., Tango, W. J., \& Booth, A. J. 2000, MNRAS, 318, 387

Eggleton, P. P., Faulkner, J., \& Flannery, B. P. 1973, A\&A, 23, 325

Farge, M. 1992, Ann. Rev. Fluid Mech., 24, 395

Gatewood, G. D., \& Gatewood, C. V. 1978, ApJ, 225, 191

Glindemann, A., Abuter, R., Carbognani, F., et al. 2000, SPIE, 4006, 2
Grevesse, N., \& Noels, A. 1993, Cosmic Abundances of the Elements, ed. N. Prantzos, E. Vangioni-Flam, \& M. Cassé, Origin and Evolution of the Elements (Cambridge: University Press)

Hanbury Brown, R., Davis, J., Allen, L. R., \& Rome, J. M. 1967, MNRAS, 137, 393

Hanbury Brown, R., Davis, J., Lake, J. W., \& Thompson, R. J. 1974a, MNRAS, 167, 475

Hanbury Brown, R., Davis, J., \& Allen, L. R. 1974b, MNRAS, 167, 121

Holberg, J. B., Barstow, M. A., Bruhweiler, F. C., Cruise, A. M., \& Penny, A. J. 1998, ApJ, 497, 935

Iglesias, C. A., \& Rogers, F. J. 1996, ApJ, 464, 943

Kamp, I., Hempel, M., \& Holweger, H. 2002, A\&A, 388, 978

Kervella, P., Coudé du Foresto, V., Glindemann, A., \& Hofmann, R. 2000, SPIE, 4006, 31

Kervella, P., Gitton, Ph., Ségransan, D., et al. 2003a, SPIE, 4838, 858

Kervella, P., Thévenin, F., Ségransan, D., et al. 2003b, A\&A, 404, 1087

Kervella, P., Ségransan, D., \& Coudé du Foresto, V. 2003c, in preparation

Kurucz, R. L. 1992, IAU Symp. 149: The Stellar Populations of Galaxies, 149, 225

Lemke, M. 1989, A\&A, 225, 125

Mihalas, D. 1978, Stellar Atmospheres, 2nd ed. (San Francisco: W. H. Freeman and Co.)

Morel, P. 1997, A\&AS, 124, 597

Morel, P., \& Thévenin, F. 2002, A\&A, 390, 611

Paquette, C., Pelletier, C., Fontaine, G., \& Michaud, G. 1986, ApJS, 61, 177

Perryman, M. A. C., Lindegren, L., Kovalevsky, J., et al. 1997, The Hipparcos Catalogue, A\&A, 323, 49

Petrov, R., Malbet, F., Richichi, A., et al. 2000, SPIE, 4006, 68

Provencal, J. L., Shipman, H. L., Høg, E., \& Tehjll, P. 1998, ApJ, 494, 759

Qiu, H. M., Zhao, G., Chen, Y. Q., \& Li, Z. W. 2001, ApJ, 548, 953

Quirrenbach, A., Mozurkewitch, D., Busher, D. F., Hummel, C. A., \& Armstrong, J. T. 1996, A\&A, 312, 160

Ribas, I., Jordi, C., \& Giménez, À. 2000, MNRAS, 318, L55

Richer, J., Michaud, G. \& Turcotte, S. 2000, ApJ, 529, 338

Royer, F., Grenier, S., Baylac, M.-O., Gómez, A. E., \& Zorec, J. 2002, A\&A, 393, 897

Schaller, G., Schaerer, D., Meynet, G., \& Maeder, A. 1992, A\&AS, 96, 269

Schlosser, W., \& Bergmann, W. 1985, Nature, 318, 45

Ségransan, D., Forveille, T., Millan-Gabet, C. P. R., \& Traub, W. A. 1999, ASP Conf. Ser., 194, 290

Ségransan, D. 2001, Ph.D. Thesis, Grenoble

Tango, W. D., \& Davis, J. 2002, MNRAS, 333, 642

Van Altena, W. F., Truan-Lian Lee, J., \& Hoffleit, E. D. 1995, General Catalogue of Trigonometric Stellar Parallaxes, Yale University

van den Bos, W. H. 1960, J. Obs., 43145

Whittet, D. C. B. 1999, MNRAS, 310, 355

Wittkowski, M., Hummel, C. A., Johnston, K. J., et al. 2001, A\&A, 377, 981

Weidemann, V. 2002, A\&A, 363, 647

Wood, M. A. 1995, in White Dwarfs, ed. D. Koester, \& K. Werner (Berlin: Springer), 41 